\title{
(Agri)culture urbaine à Cuba : émergence et impacts de l'agriculture urbaine à Cuba
}

Auteur $=$ Nom $:$ ARGAILLOT $/$ Prénom $:$ Janice

Résumé : Ce travail propose d'analyser l'essor de l'agriculture urbaine à Cuba. En effet, à partir une nécessité induite par la crise subie par l'île - du fait de l'effondrement de l'URSS et $\mathrm{du}$ renforcement $\mathrm{du}$ blocus états-unien- Cuba a construit un modèle d'agriculture internationalement reconnu, et ce pour plusieurs raisons. Nous nous intéresserons donc aux causes de la mise en place du système d'agriculture urbaine à Cuba, avant d'analyser ses différentes formes puis ses répercussions dans la vie sociale et culturelle des citadins.

Abstract : This article aims to analyze the development of urban agriculture in Cuba. Indeed, from a necessity leaded by the crisis faced by the Island -due to the URSS collapse and the strengthening of the United States blockade - Cuba has built a model of agriculture internationally recognized, for many reasons. We will then take an interest in the causes of the urban agriculture raising in Cuba, before analyzing its different forms and repercussions in the social and cultural life of citizens.

Resumen : Este trabajo propone un análisis del auge de la agricultura urbana en Cuba. En efecto, a partir de una necesidad inducida por la crisis enfrentada por la Isla -por el colapso de la URSS y el refuerzo del bloqueo estadounidense- Cuba ha construido un modelo de agricultura internacionalmente reconocido, por varias razones. Nos interesaremos pues por las causas de la puesta en marche del sistema de agricultura urbana en Cuba, antes de analizar sus diferentes formas y repercusiones en la vida social y cultural de los ciudadanos.

Mots-clés : Cuba, agriculture, ville, Période spéciale, société

Keywords : Cuba, agriculture, city, Special period, society

Palabras claves : Cuba, agricultura, ciudad, Período especial, sociedad 


\section{(Agri)culture urbaine à Cuba : émergence et impacts de l'agriculture urbaine à Cuba}

La plus grande île de la Caraïbe s'est traditionnellement élevée en un espace agricole de monoproduction. Néanmoins, on ne peut que constater qu' « à Cuba comme ailleurs, surtout dans le Tiers monde, l'essor des villes est un phénomène de notre siècle [...] » (Roux, 1997). Ainsi, les mutations qui se sont opérées dans l'Île et qui ont poussé la population vers les grandes agglomérations, ajoutées aux difficultés économiques engendrées par la disparition de l'allié soviétique, ont nécessité une adaptation constante de l'espace urbain. En effet, il a fallu trouver des réponses à la fois rapides et durables aux besoins des nouveaux arrivants, notamment sur le plan alimentaire. L'agriculture urbaine s'est imposée comme l'une d'entre elles, mais il faut dire qu'elle ne contribue pas uniquement à la subsistance physique des villes, dans la mesure où elle est également devenue un élément moteur de la vie culturelle et sociale des citadins cubains ${ }^{1}$.

Ainsi, nous souhaitons dans un premier temps mettre en lumière les événements ayant poussé les autorités et la population cubaine à se tourner vers l'agriculture urbaine. En effet, cette réorientation de l'espace urbain et ce nouveau visage donné à l'agriculture sont le corollaire d'une crise violente et globale subie par les Cubains, et il est nécessaire de se demander de quelle façon ces derniers perçoivent ce phénomène relativement récent.

Dans un deuxième temps, nous nous intéresserons aux diverses formes de l'agriculture urbaine cubaine. En effet, l'agriculture pratiquée au sein des grandes agglomérations cubaines n'est pas monolithique ; elle implique au contraire implique différents acteurs, s'insinue dans de multiples espaces, et produit une large gamme de denrées.

Enfin, nous tenterons de comprendre les répercussions de ce type d'agriculture sur la culture et l'identité des personnes la pratiquant, mais aussi sur la géographie culturelle des villes, puisqu'elle peut se révéler être un facteur positif pour l'environnement, les liens sociaux et les pratiques culturelles.

I/Essor de l'agriculture urbaine dans la Cuba contemporaine

\footnotetext{
${ }^{1}$ Notre étude sera principalement centrée sur la capitale cubaine, véritable laboratoire pour l'agriculture urbaine et reflet de l'orientation politique de l'ensemble du pays, même si d'autres villes pourront être abordées, du fait de leur(s) particularité(s).
} 


\section{A. La chute de l'URSS}

Il faut avant tout rappeler l'ampleur de la crise économique subie par l'île depuis l'effondrement du bloc communiste, qui s'est répercutée dans tous les secteurs productifs ainsi que dans tous les domaines de la vie privée des Cubains, et a débouché sur la « Période spéciale en temps de paix ». Cette période, dans laquelle l'Île entra concrètement au début des années 1990 et qui fut officiellement décrétée par le gouvernement cubain à l'été 1991 -dont la fin n'a toujours pas été annoncée à ce jour- fut en réalité constituée d'une série de mesure d'austérité visant à préserver l'économie de l'Île, afin de maintenir une cohésion sociale et en conséquence de sauver le système révolutionnaire.

Durant la Période spéciale, assurer l'approvisionnement minimum en denrées alimentaires des villes devint une priorité, puisque le bloc socialiste assurait $66 \%$ des besoins de l'Île dans ce domaine (Farinelli, 2008). Mais avant même la disparition de l'Union Soviétique, Cuba avait mis en place un plan d'urgence permettant de réaliser des économies, et en conséquence de sauvegarder les acquis de la Révolution : «A La Havane, [...] on favorise l'agriculture urbaine comme moyen de combler les graves pénuries alimentaires qui frappe le pays depuis « la période spéciale en temps de paix » qui a débuté en 1989 avec la diminution draconienne de l'aide soviétique et du commerce avec l'URSS »(Moscow, 2000). Ainsi, il convenait d'assurer la subsistance de la population, désormais soumise à la " libreta » (carnet de rationnement), et ceci tout particulièrement en ville. Néanmoins, la production agricole « traditionnelle » devenait insuffisante à l'alimentation des grandes villes qui avaient absorbé un fort exode rural, et au sein desquelles « les anciens ruraux n’ont pas les revenus monétaires suffisants pour acheter leur nourriture [...] » (Maréchal, 2010) : « La capitale cubaine, La Havane, abrite $20 \%$ de la population de l'île, soit quelque 2,5 millions d'habitants. Nourrir cette population compte [...] parmi les priorités » (Madeley, 2002).

Mais l'effondrement de l'URSS constitua bien un choc pour l'Île, et ce à différents niveaux. Sur le plan économique, il faut souligner qu' « au cours de l'année précédent l'éclatement final de l'URSS, la balance commerciale de Cuba a subi une perte de $80 \%$ et a vu sa production agricole diminuer de moitié en raison de la disparition soudaine de presque 1,3 millions de tonnes d'engrais » (Annuaire du PNUE, 2009). Ainsi, malgré les efforts de l'État, 
la situation se dégrada fortement : « Le premier semestre de l'année 1993 se révèle catastrophique dans tous les domaines. Les pénuries sont particulièrement graves dans la production électrique, les transports et l'alimentation de la population urbaine, en particulier dans la capitale et les grandes villes où sévit une disette rampante, aggravée par une épidémie de neuropathie liée à la malnutrition » (Douzant Rosenfeld, 1996).

Il s'agissait donc réellement d'assurer la survie de la population. Les autorités se virent ainsi obligées à repenser le rôle des acteurs de la chaîne d'approvisionnement alimentaire l'effondrement de l'URSS fut en cela le « catalyseur à un changement de politique radical »(Annuaire du PNUE, 2009) - et la taille même des unités de production fut questionnée. On observe d'ailleurs depuis le début des années 1990 la tendance de l'agriculture cubaine à aller de plus en plus vers le local et le particulier, ainsi que l'autonomie croissante des producteurs : « La mesure la plus spectaculaire est la décision de démanteler les fermes d'État pour arrêter de les subventionner à perte. Il s'agit de remplacer les diverses unités de base de production au sein des grandes exploitations par des unités autonomes de taille moyenne sous forme de coopératives autogérées [...]»(Douzant Rosenfeld, 1996). Partant, il convient de dire que la disparition de l'URSS a supposé une réorganisation politique et économique de l'agriculture cubaine.

B. L'embargo états-unien

À la pénurie interne s'ajoutait l'embargo états-unien, qui donna sans le vouloir l'impulsion décisive au développement de l'agriculture urbaine à Cuba : " L'agriculture urbaine contemporaine a commencé par se développer à Cuba du fait de l'embargo » (Lepage, 2009). En effet, la Période spéciale fut un moment jugé opportun par le gouvernement états-unien à la chute du « régime » cubain. De la sorte, les États-Unis ont accru leur politique d'isolement de l'Île (notamment par le biais des lois Torricelli en 1992 et Helms-Burton en 1996), qui a vu ses importations chuter de manière drastique : «Estados Unidos hizo aún más estricto su bloqueo contra Cuba, lo cual creó dificultades adicionales para la isla. Cuba vio gravemente amenazado su acceso a los alimentos, y sus importaciones en este aspecto cayeron a la mitad. De ahí que el consumo de calorías disminuyera en un 22\%, el de proteínas en un 36\% y el de grasas en un 65\% » (Programme des Nations Unies pour le Développement, 2002). 
On comprend donc aisément que l'État cubain ait soutenu une politique de développement de l'agriculture urbaine face à l'impossibilité d'importer un grand nombre de denrées : «Le gouvernement a créé un programme qui a transformé en jardins les nombreux espaces vacants de La Havane. Certains quartiers vont même jusqu'à produire $30 \%$ de leur alimentation » (Lepage, 2009). Cette politique a ainsi été couronnée de succès, puisqu'on a vu fleurir de nombreux jardins potagers au sein même de la capitale : « Avant 1989, on n'avait pratiquement jamais entendu parler d'agriculture urbaine ; en 1998, grâce à l'appui de l'État, $30 \%$ du territoire disponible à La Havane était occupé par des « jardins » officiellement reconnus et entretenus par plus de 30000 personnes » (Madeley, 2002).

L'agriculture urbaine n'est ainsi pas une constante depuis le début de la Révolution. Il s'agit plutôt d'un changement de cap dû à la situation économique du pays : « Par nécessité, Cuba a décidé de s'orienter vers les vieilles méthodes : agriculture biologique, traction animale, amendement naturel des sols, horticulture de proximité (puisque les transports manquaient)... [...] L'agriculture de proximité était développée par la distribution de centaine de terrains vacants à qui voulait les cultiver, et par l'incitation à cultiver partout où l'on pouvait : dans les patios, sur les terrasses des immeubles -dans des pots, des containers ou des pneus- des coopératives agricoles étaient créées, de même qu'un réseau des boutiques de graines et d'outillage ou des consultants donnaient des conseils aux utilisateurs » (Farinelli, 2008). On ne peut nier ici l'échec du plan états-unien, puisque l'embargo n'a pas permis la chute du « régime cubain », et qu'il a en outre favorisé une certaine cohésion sociale face à l'adversité. Ainsi, il faut dire que le jardinage, cette nouvelle pratique qui s'est imposée comme une nécessité, n'est pas nécessairement perçue comme un mal par les citadins. Jardiner serait même pour certains un acte révolutionnaire, en tous les cas un « moyen de contribuer à la révolution »(Moscow, 2000). En somme, aider à la production alimentaire du pays reviendrait dans l'esprit de certains à combattre les pénuries et l'embargo états-unien, autrement dit à poursuivre la lutte révolutionnaire ainsi que la politique d'indépendance de l'Île vis-à-vis du Voisin du nord. On relève donc que certains citadins ne voient pas dans le fait de jardiner une « obligation » qui serait la résultante des manquements du système 
révolutionnaire ; la faute incombe uniquement pour une partie de la population à la politique hostile des États-Unis.

II/Une agriculture urbaine, différentes formes

Si l'urbain relie les pratiques agricoles étudiées ici, il faut préciser que l'agriculture urbaine cubaine est diverse, et prend différentes formes.

\section{A-Espaces}

Dans un premier temps, il faut souligner que les espaces publics ont en partie été réquisitionnés par les autorités et convertis en lieux de production agricole. Ainsi, les jardins personnels sont cultivés sur « des terrains privés ou des terres de l'État que les jardiniers peuvent utiliser sans frais. Selon les estimations, il en existerait plus de 26000 à La Havane » (Moscow, 2000). Le fait de mettre à disposition des citadins les espaces publics afin de leur garantir un approvisionnement minimum n'est pas anodin. Il contribue effectivement à diffuser l'idée d'un État soucieux de faire des sacrifices pour son peuple. Autrement dit, il ne s'est pas agi uniquement de nourrir la population, mais également de poursuivre métaphoriquement et physiquement la lutte que suppose la Révolution.

On a ainsi vu se multiplier les autoconsumos, jardins potagers de subsistance, le plus souvent géré par une entité administrative. Les autorités cubaines soutiennent ainsi l'agriculture urbaine, et dans une certaine mesure, tentent même de la contrôler -certains diront de la surveiller. Il faut néanmoins préciser que dans les fermes d'État (« fincas estatales »), 50\% des bénéfices sont redistribués aux travailleurs (Cosecha urbana, 2004).

En parallèle, en donnant son aval à la production personnelle d'aliments - les marchés paysans ont été (ré)instaurés en 1994, alors qu'ils avaient été interdits en 1986- l'État cubain a, sans le vouloir, consolidé le marché noir. Il est important de comprendre que l'agriculture urbaine est un phénomène global, et d'insister sur le fait qu'une partie de la production reste contrôlée par l'État, alors qu'une autre est le fait de citadins indépendants, qui cultivent leur propriété : «À La Havane, tous les espaces disponibles, y compris les toitures et les balcons, 
sont affectés à la production de produits alimentaires » (Programme des Nations Unies pour l'Environnement, 2002).

Dès 1989, les centres de restauration collective connurent des bouleversements : «[Les cantines urbaines qui] restent ouvertes se dotent d'un potager en travail collectif, comme certaines parcelles d'agriculture urbaine se glissent dans les interstices du tissu urbain havanais ou des grandes villes »(Roux, 1997). On peut ainsi affirmer que la réponse du gouvernement cubain à la crise a été à la fois rapide et progressive, puisque les espaces urbains étaient peu à peu « conquis » par l'agriculture au fur et à mesure de l'accentuation de la crise.

De cette façon, les « terrains vagues », « lots abandonnés » ou « décharges » ont été convertis en jardins (Moscow, 2000), ce qui contribue à la diffusion d'un sentiment de plus grande sécurité -puisque des terrains jusqu'alors désertés et qui étaient devenus des zones « informelles » ont été transformés en potagers-, tout en contribuant à l'embellissement de la ville. En ce sens, l'agriculture urbaine constitue un facteur d'amélioration du lieu de vie, donne un nouveau sens à des espaces inutilisés, et s'apparente donc à un renouveau du cadre urbain.

Par ailleurs, si l'agriculture urbaine s'est dans un premier temps cantonnée aux espaces « abandonnés » de la ville, elle a au sens propre et au fil du temps pris une place de plus en plus importante dans la vie quotidienne des citadins cubains : « La creación de un sistema de agricultura urbana fue un componente clave para construir un nuevo sistema alimentario autosuficiente y sostenible, y para acercar más a consumidores y productores. Este esfuerzo fue inicialmente una respuesta popular a la escasez de comida, por la cual algunos residentes urbanos cultivaron solares abandonados a principios de los 1990. Pero pronto el gobierno cubano vio el potencial que ofrecía la agricultura urbana para aliviar la crisis alimentaria. Escuelas, instituciones y lugares de trabajo empezaron a producir alimento en sus tierras. Los jardines urbanos brotaron por toda la ciudad de La Habana, sobre todo en forma de jardines en las casas, en los centros cívicos y en solares vacíos » (Fernández, 2008). On voit ici que le gouvernement cubain s'est finalement rangé à une pratique impulsée par les citadins eux- 
mêmes, et qu'il a contribué à la développer en lui offrant de nouveaux espaces, parfois perçus comme le centre d'une culture plus « intellectuelle», telles que les écoles.

Il convient encore de souligner que le Parc Métropolitain de La Havane est au cœur d'une tentative d'" élaborer une forme d'agriculture urbaine, globale, écologique et autosuffisante »(Rodriguez, 2000 : 90). Cela peut paraitre incongru (Rodriguez, 2000), le métropolitain semblant a priori s'opposer au rural et à l'agricole, mais les différents problèmes liés à l'économie et l'écologie que connaît l'Île appellent des réponses globales et rapides, qui permettent en outre une meilleure communication entre deux mondes.

Il est difficile de faire une estimation exacte de la superficie cultivée, le terme « urbain » n'ayant pas la même acception par tous les observateurs. De nombreux chiffres circulent ainsi sur internet ; malgré tout, on peut dire que l'agriculture urbaine a bien envahi l'ensemble de la capitale cubaine. Il est indéniable qu' « il y en a beaucoup plus en périphérie qu'au centre de la ville, mais les potagers se trouvent un peu partout » (Moscow, 2000) à La Havane. On observe ici que depuis la périphérie, l'agriculture urbaine a bien gagné le centre, le cœur de la ville.

Si elle offre aux citadins un moyen de subsistance, cette agriculture est également un moyen pour l'Île de projeter une image positive d'elle-même sur la scène internationale, puisque les acteurs de la permaculture cubaine sont invités à de nombreuses conférences de par le monde. Ainsi, l'expérience et les réalisations cubaines sont-elles un moyen pour le gouvernement révolutionnaire de donner à voir une autre image que celle de pays « miséreux » que l'on a parfois voulu lui accoler, et devient un lien entre l'île et d'autres pays, brisant par là même l'anathème dont elle a pu être victime. À titre d'exemple, la ville de Cuenca, en Équateur, a bénéficié de l'aide de la ville de La Havane lors de la mise en place de son projet d'agriculture urbaine en 1998 (Cosecha Urbana, 2004).

L'agriculture urbaine est encore un thème de débat et de réflexion, notamment pour les pays économiquement «sous-développés », dont Cuba s'est souvent posée en défenseur, voire en fer de lance. En effet, de nos jours, l'agriculture urbaine n'est plus uniquement un moyen de pallier une crise ponctuelle ; elle est au contraire de plus en plus perçue comme un moyen de 
faire face à des difficultés globales et latentes, c'est-à-dire à une crise des systèmes politiques et sociaux.

Quoi qu'il en soit, on ne peut nier l'importance acquise en peu de temps par l'agriculture urbaine pour Cuba : «A doce años establecida, la agricultura urbana ocupa más de 1500 hectáreas en la trama urbana de la ciudad de La Habana y asegura el uso productivo, con incremento de masa verde, de un número considerable de espacios urbanos » (Cruz, 2004). Ce type d'agriculture s'est bien imposé dans toute la ville, et n'est plus clairement limité. Son impact est donc lui aussi global, d'autant plus qu'outre les lieux de production, il faut prendre en compte les lieux de commercialisation : «Existen diversos lugares donde se comercializan los productos de AU [agricultura urbana], como : placitas normadas, puntos de venta, mercados agropecuarios estatales, mercados agropecuarios, de oferta y demanda, contratos directos con círculos infantiles, escuelas, hospitales y otros, restaurantes vegetarianos, ventas al turismo y empresas de flores " (Cosecha urbana, 2004). Ainsi, derrière l'aspect commercial, l'agriculture urbaine implique différents acteurs, et dévoile en creux de nombreuses problématiques.

\section{B-Acteurs}

Le gouvernement, nous l'avons vu, a joué un rôle primordial dans l'essor de l'agriculture urbaine. D'aucuns vont jusqu'à dire qu'il en est le soutien et le moteur, ce dont peu d'autres États peuvent se prévaloir : « Le rôle du gouvernement cubain dans la promotion et le soutien de l'agriculture urbaine est unique au monde et compte sûrement pour beaucoup dans la réussite du programme » (Moscow, 2000).

Le Ministère de l'Agriculture a promu l'agriculture urbaine dès 1991 (Moscow, 2000). Ce même Ministère a créé en 1994 le Département d'agriculture urbaine, dans le but d'aider la population à acquérir des techniques agricoles, et lui a fourni le matériel nécessaire à son bon

développement (Fernández, 2008). La création de ce Département montre à elle seule l'importance que revêt l'agriculture urbaine pour Cuba, mais également la pleine conscience des autorités de la nécessité de la développer. Cela est à tel point vrai qu'en l'an 2000, 
l'agriculture urbaine a été intégrée au Plan de Ordenamiento Territorial de la ville de La Havane (Cruz, 2004).

Outre les institutions, les grandes figures de la Révolution ont également appuyé le développement de l'agriculture urbaine. Raúl Castro lui-même enjoignait les Forces Armées à participer aux processus d'autoconsommation, et son frère Fidel appuyait sans réserve « le modèle alternatif » que constituait l'agriculture urbaine (Programme des Nations Unies pour le Développement, 2002).

Si l'agriculture urbaine a envahi la totalité de l'espace urbain, elle est également promue par l'ensemble du système politique, les échelons nationaux et provinciaux se complétant afin de l'impulser. La ville de Cienfuegos en est l'un des exemples : « La trayectoria de Cienfuegos, capital de la provincia de Camagüey, [...] con 163 mil habitantes, parece haber sido más exitosa al encarar la crisis nacional que se presentó desde comienzos de los años noventa, primero mediante subsidios temporarios y asistencia social, y luego complementando las acciones del gobierno nacional con un conjunto de programas propios como el Programa de Agricultura Urbana y el Programa de Desarrollo Integral del Municipio. Ambos programas son operados en coordinación con los niveles jurisdiccionales nacional y provincial y la participación de las unidades de organización de la población, tanto al nivel municipal como en el territorio urbano » (MacDonald, 2005). En complétant très rapidement les programmes nationaux par des aides et programmes spécifiques à la région, Cienfuegos a connu un essor rapide de sa production agricole urbaine, lui permettant même en 2002 de produire 63,431 tonnes de légumes, graines et tubercules (Cosecha urbana, 2004).

Les provinces et les villes ont été épaulées dans le travail de diffusion des connaissances techniques par des « agents de vulgarisation agricole » (Moscow, 2000). En somme, on a donné à la population les moyens de parfaire ses connaissances et les outils pour maîtriser une agriculture spécifique, à laquelle elle n'était pas habituée. En conséquence sans doute, il faut souligner que la population s'est impliquée. En participant aux formations offertes, les citadins cubains ont répondu favorablement à l'initiative gouvernementale. 
D’une façon générale, les femmes sont considérées comme les principales actrices de l'agriculture urbaine. Néanmoins, et même si la crise subie par l'Île est bien totale et globale (elle touche tous les secteurs, toutes les catégories d'âge et socioprofessionnelles, et implique donc la collectivité), il semble que « les responsabilités domestiques des femmes [à tout le moins au début de la période spéciale en temps de paix] ne leur laissaient pas assez de temps pour faire du jardinage » (Moscow, 2000) ; les hommes seraient donc les principaux acteurs de l'agriculture urbaine cubaine.

Certaines fondations et certains centres d'études participent également de façon active à la promotion de l'agriculture urbaine. Ainsi, la Fondation Antonio Núñez Jimenez défend notamment la permaculture, et l'une de ses publications, ILE, Anuario de Ecología, Cultura y Sociedad, montre par son titre même l'importance de lier l'humain et toutes les cultures, y compris l'agricole.

Mais il faut également dire que les « dissidents » ont fait de la politique d' « autoconsumo » une tribune pour leurs revendications. De la sorte, le parti « Cuba Independiente y Democrática » (CID) a organisé diverses réunions afin de promouvoir des projets portant sur ce sujet. Il faut rappeler les liens du CID avec les États-Unis, et se demander en conséquence si le fait de revendiquer l'autonomie alimentaire ne cache pas la volonté d'aller vers une « liberté » avant tout politique.

\section{C-Produits}

On observe que la production des espaces agricoles urbains est diversifiée, tout comme les modes de production. En 1995, on dénombrait 1613 " organopónicos 》 (coopératives horticoles au sein desquelles le plafond de prix des produits vendus est contrôlé par l'État), 429 « huertas intensivas », et 26604 « huertas populares » à Cuba (ElHage Sciabbala, Hattam, 2003). Le poids des organopónicos dans la production agricole urbaine est indéniable : « The organopónicos of Cuba have become the prime example for other countries to gain food security. In Havana alone there are 200 urban farms producing half the city's fruit and vegetables. Havana has one-fifth of Cuba's population. Food can be bought for low prices 
directly from these gardens. [...] Manly do poly-cropping, mixed farming of animals, crops, vegetables and fruit claimed to be 25 times more productive than farming each separately" (Houbein, 2011).

En 1994, les potagers organiques urbains produisirent 4000 tonnes d'aliments (ElHage Sciabbala, Hattam, 2003). On constate donc qu'il ne s'agit pas d'un phénomène mineur ou périphérique. L'agriculture urbaine est même devenue un pilier essentiel de l'économie par l'étendue et la force de sa production : «En 1999, la agricultura orgánica urbana no certificada (en huertas domésticas, canteros elevados y huertas intensivas) produjo el 65 por ciento del arroz del país, el 46 por ciento de las verduras frescas, el 38 por ciento de las frutas no cítricas, el 13 por ciento de las raíces, tubérculos y plátanos y el 6 por ciento de la producción de huevos" (ElHage Sciabbala, Hattam, 2003)². Ainsi, l'agriculture urbaine cubaine produit essentiellement des fruits et des légumes, mais également du riz, aliment qui constitue la base de l'alimentation cubaine.

En outre, les agriculteurs urbains développent depuis plusieurs années de petits élevages d'animaux - principalement de basse-cour- fournissant ainsi volailles et œufs à la population urbaine. À cela s'ajoute la culture maraîchère, également importante dans les zones urbaines. On voit ici que l'agriculture qui s'est développée dans les villes cubaines fournit un large éventail de produits, et que même si les producteurs urbains cultivent ou élèvent avant tout pour leur propre consommation, il n'est plus possible, au contraire du début des années 1990, d'évoquer une « disette ».

III/Quel(s) impact(s) sur la population, son environnement et sa culture ?

L'agriculture urbaine suppose donc des transformations, mais également des bénéfices multiples : « Les avantages des jardins peuvent également s'étendre aux quartiers avoisinants. On relève cinq types d'avantages : augmentation des denrées alimentaires disponibles, contribution au pays, embellissement des quartiers, amélioration de la sécurité et amélioration de l'écologie urbaine » (Moscow, 2000).

\footnotetext{
2 Pour la même année, certaines sources donnent des chiffres légèrement différents : l'agriculture urbaine aurait produit 800000 tonnes de denrées, et notamment du riz (65\% de la récolte nationale), des fruits $(43 \%)$, des racines et tubercules (12\%) d'après le Programme des Nations Unies pour le Développement (2002 : 148).
} 
A-Écologie

L'environnement et l'écologie sont deux facteurs primordiaux pour la production agricole mondiale, car la nécessité de produire à tout prix ne prime plus sur le développement durable -à tout le moins dans les discours officiels. Le premier avantage de la production agricole urbaine est donc de lier approvisionnement alimentaire et respect de l'environnement : « Ainsi, la production alimentaire est très transparente. Elle est également saine pour l'environnement, car elle nécessite peu de transport et d'entreposage » (Moscow, 2000). En produisant « sur place », le consommateur bénéficie d'une certaine traçabilité des produits, qui ne nécessitent que peu de transport, ce qui réduit l'émission de pollutions.

Certains déchets sont transformés en compost, ce qui constitue en outre un bénéfice économique. De la sorte, l'agriculture urbaine permet le recyclage des déchets organiques de la ville. Elle permet également de sensibiliser la population quant à la protection de l'environnement, sensibilisation qui est encore une fois appuyée par des associations et le gouvernement : « En el año 1992, se fundó la Asociación Cubana de Agricultura Orgánica y desde entonces desempeña un importante papel en la promoción de la agricultura orgánica, desarrolla campañas de concientización, implementa proyectos y crea modelos pilotos. Como consecuencia, se han fortalecido las relaciones entre los Ministerios gubernamentales con respecto a la agricultura orgánica y aumentó la participación de las organizaciones y cooperativas de agricultores en todo el país » (ElHage Sciabbala, Hattam, 2003).

Ainsi, l'écologie se convertit peu à peu en un thème central de l'agriculture urbaine. Finalement, l'une des «[...] caractéristiques importantes de l'agriculture durable cubaine [est] : dans les zones urbaines, trois types de potagers intensifs cultivés en biologique : des jardins pour assurer l'autosuffisance des écoles et des lieux de travail (« autoconsumos »), des jardins à plates-bandes en billon (« organopónicos ») et des jardins communautaires intensifs (huertos intensivos) »(ONU, 2002). L'aspect « biologique » est primordial, puisque le succès et les avancées sont tels, que l'on peut désormais lire qu'en ce qui concerne l'agriculture urbaine mondiale, « la réussite la plus importante, et pour partie la plus formalisée, est celle 
de Cuba dont le FAO s'efforce de faire partager l'expérience dans tous les pays où l'urbanisation galopante pose problème [...]. » (Maréchal, 2010). Ainsi, les Cubains sont présentés comme les « leaders dans les domaines de la préservation des sols, des méthodes d'agriculture organique, des biopesticides ou du vermicompostage » (Annuaire du PNUE, 2009).

L'agriculture urbaine cubaine est donc essentiellement, pour ne pas dire exclusivement, organique et biologique, les problèmes économiques empêchant l'achat d'engrais chimiques (un décret publié en 1996 interdit d'ailleurs l'agriculture non biologique à La Havane). On a ainsi remis au goût du jour les bio-fertilisants, et promu une agriculture en lien avec le monde animal (utilisation de vers de terre, d'animaux de pâturage...). Et à ce sujet, certains petits exploitants urbains, qui travaillent sans produits chimiques ni machines, se sont montrés «plus efficaces que les unités de production à grande échelle » : «[...] se suele argumentar la necesidad de la eficiencia de la producción a gran escala para producir suficientes alimentos. Sin embargo, en Cuba encontramos pequeños productores y agricultores urbanos en la vanguardia del proceso de recuperación de la depresión económica en los 90, que sin maquinaria e insumos químicos han demostrado ser más eficientes que las unidades de producción a gran escala » (Botella Rodríguez, 2007).

Il faut même dire que l'agriculture urbaine cubaine, orientée vers l'écologie -en premier lieu pour des raisons, insistons sur ce point, économiques- a induit un véritable bouleversement dans les «traditions » : « La agricultura sostenible no es la norma en América Latina, y la agroecología no tiene gran influencia en las principales agendas de la investigación. [...] La experiencia de Cuba [...] indica que cuando el campesino a campesino es empleado en un contexto político que promueve la agroecología y el desarrollo conducido por el campesino, los agricultores y sus organizaciones rápidamente hacen que la agricultura sostenible sea la norma en lugar de la excepción. El movimiento campesino a campesino comenzó primero con grupos de agricultura urbana en múltiples cinturones verdes alrededor de La Habana [...] » (Knox et Meinzen-Dick, 2006). Il s'agit bien ici d'une mutation, l'exception d'hier se convertissant en la norme d'aujourd'hui. 
On présente l'agriculture urbaine comme une façon de résoudre les problèmes actuels, et, en l'alliant au développement durable, comme une manière d'assurer de bonnes conditions de vie aux générations futures (Cruz, 2005). En effet, il ne faut pas oublier qu'au cœur de toutes les difficultés se trouve finalement la population cubaine, dont la vie sociale, quotidienne et culturelle s'est également vu transformée par le surgissement de l'agriculture urbaine.

B-Vie sociale et culturelle

La vie sociale a connu des évolutions grâce à l'agriculture urbaine, puisque l'on peut parler d'un certain rapprochement entre les habitants d'un même quartier. L'agriculture urbaine resserre donc dans une certaine mesure les liens sociaux, non seulement au sein des villes, mais également entre espace urbain et rural : Le « [...] rapprochement entre le lieu de production et le lieu de consommation conduit à une atténuation de la séparation villecampagne, sous l'effet de la renaturation des villes [...]»(Lepage, 2009). L'agriculture urbaine a donc impacté sur le quotidien des citadins en modifiant le visage « traditionnel » de la ville : «[...] les animaux de ferme sont beaucoup plus présents à La Havane que dans bien des capitales ; on les garde souvent près de son domicile pour les protéger contre les voleurs » (Moscow, 2000). En somme, l'espace urbain a été transformé, tout comme son usage classique, et on observe une véritable redéfinition de la ville en tant que lieu de vie, ainsi que de ses frontières : «Esta nueva función urbana está interactuando con la agricultura rural, no solo para complementarse en la disponibilidad de productos agrícolas para la población, sino y de forma muy importante, en la transformación de las relaciones campo-ciudad y en las debilidades de la cultura agraria heredadas de la época colonial y reafirmadas durante este siglo $[\ldots] »($ Cruz, 2004).

Malgré tout, il faut souligner que la population a dû s'adapter aux nouvelles conditions de production, puisque l'Île n'était traditionnellement pas un pays de culture potagère, et que le déplacement de l'agriculture vers la ville suppose sans doute un " choc ». Il ne faudrait d'ailleurs pas sombrer dans l'écueil de transposer le modèle rural à l'espace urbain (Cruz, 2004). 
Par ailleurs, l'agriculture urbaine a également contribué à la création d'une société à deux vitesses, et a parfois exacerbé les tensions et la jalousie entre les personnes ayant la possibilité de cultiver un lopin de terre et les autres. De la même façon, il convient de noter que certaines zones de La Havane reçoivent un soutien financier du Ministère de l'Agriculture dans le but de développer l'agriculture urbaine, tandis que d'autres ne s'appuient que sur « les militants du quartier » (Moscow, 2000).

En parallèle, il faut reconnaître que l'agriculture urbaine a favorisé la création d'emplois : « La principal motivación de Cuba fue evitar el desabastecimiento de alimentos, pero su apoyo a la agricultura en las ciudades ha constituido además una sabia inversión en empleo y en prevención de crisis. La agricultura urbana ha creado en Cuba 160.000 puestos de trabajo, incluyendo trabajadores agrícolas, albañiles, vendedores y personas empleadas en el secado de hierbas y la producción de abono » (Halweil, Nierenberg, 2007). Autrement dit, ce nouveau type d'agriculture a permis, dans une certaine mesure, de lutter contre un chômage que l'on disait endigué, mais qui avait reparu avec la crise.

Bien évidemment, « la culture et l'élevage [contribuent] à augmenter de façon significative la quantité d'aliments à la disposition des familles des jardiniers et la qualité de ces aliments » (Moscow, 2000). La diète urbaine a en ce sens connu une évolution positive, et si les Cubains continuent à conjuguer le verbe « resolver » à tous les temps, l'agriculture urbaine leur a tout de même offert un certain répit, une certaine tranquillité d'esprit concernant leur approvisionnement en denrées alimentaires.

L'agriculture urbaine participe également pleinement de la vie économique non seulement du pays, mais également de ses habitants : «Les jardins ont également un effet marqué sur les budgets des ménages : ils contribuent à réduire les dépenses alimentaires hebdomadaires et les jardiniers peuvent gagner de l'argent en vendant leurs produits maraîchers » (Moscow, 2000). Pour le dire autrement, les bénéfices financiers induits par l'agriculture urbaine ne contribuent pas uniquement à la préservation des institutions politiques ; ils sont également primordiaux pour les citadins, dans leur quotidien. 
Conséquemment, des études ont démontré que pour certains jardiniers urbains, le jardin « [donne] l'impression d'exercer une plus grande maîtrise sur leur vie » (Moscow, 2000). La crise qui a frappé de plein fouet tout le pays paraissait être une déferlante incontrôlable, mais le jardinage urbain s'est imposé comme un moyen très concret d'y faire face. Les personnes qui consacrent une partie de leur temps à cultiver des potagers semblent donc regagner une certaine confiance en eux, se percevant comme les principaux acteurs de leur destin, et ne plaçant pas tous leurs espoirs en un « État providence ».

En outre, le jardinage leur apparaît comme « divertissant », et leur jardin comme « un endroit attrayant » (Moscow, 2000). Jardiner est parfois pour eux synonyme de « réduction du stress, satisfaction spirituelle, plaisir et esthétisme » (Moscow, 2000). Il ne s'agit donc plus uniquement de cultiver pour subsister, dans la mesure où le jardinage s'est converti en un plaisir, et peut-être en un moyen de s'échapper des réalités difficiles du quotidien.

En outre, l'agriculture urbaine à Cuba n'est pas dénuée d'une dimension « communautaire » (Cruz, $2005: 328)$. Tout en mettant en contact plus de 15000 producteurs (Cruz, 2004), elle est au centre de projets qui ne peuvent être réalisés qu'en concertation avec tous les acteurs de la ville, et est également un moteur d' « inclusion sociale » : "Pero además, la agricultura urbana propicia espacios de acción conjunta entre población, gobiernos locales y sociedad civil, es promotora de fuentes de empleo técnico y productivo y es un componente modesto de economía comunitaria. Por último, [...] la agricultura urbana genera la inclusión social de sectores vulnerables [...] »(Cruz, 2005). En somme, l'agriculture urbaine cubaine offre un espace de discussion et d'échanges, et permet à tous les secteurs de la société d'entrer en communication; elle devient donc un facteur de réduction de l'exclusion sociale en valorisant à la fois l'identité individuelle et collective.

L'agriculture urbaine offre en outre un espace presque onirique, une fenêtre ouverte sur un futur que l'on souhaiterait meilleur : «On imagine, aujourd'hui, la création de fermes urbaines coopératives, la création d'espaces de nature en ville, la relance de la biodiversité apportant des emplois donnant priorité aux populations locales exclues du système, permettant ainsi la production et la livraison d'une alimentation saine et de proximité » (Lepage, 2009). 
Si la vie en communauté a été touchée par l'instauration de l'agriculture urbaine, la culture a également connu des transformations. En effet, la ville est avant toute chose une production humaine. Ce sont donc bien les citadins, qui construisent et vivent la ville, qui impulsent sa gestion quotidienne. Dès lors que l'on évoque l'humain, l'identité et la culture affleurent rapidement. Et l'on ne peut que souligner que l'agriculture urbaine a un impact sur cette culture. Elle favorise effectivement les actions locales, et en conséquence permet une plus grande implication des populations dans des projets communs (Cruz, 2005).

Ce type d'agriculture bouleverse également les schémas établis, et demande aux habitants des grandes agglomérations certaines capacités d'adaptation : «Pero también es necesario que se modifiquen debilidades culturales del ciudadano, su condición de consumidor, generador de deshechos, con una visión distante de lo rural [...]»(Cruz, 2005). Le fait de ne plus être seulement une activité productive et commerciale pérennise sans doute l'agriculture urbaine, et l'ancre bien dans une culture à la fois locale et nationale.

\section{Conclusion :}

De nombreux observateurs s'accordent à dire que l'expérience cubaine est un exemple pour tous les pays (Botella Rodríguez, 2007). En effet, d'une expérience nécessaire à la survie économique du pays, s'est développée une nouvelle forme d'agriculture, plus respectueuse des traditions, de l'environnement et des Hommes.

Il convient de noter que La Havane est même un exemple de réussite de la mise en place de projets d'agriculture urbaine : «En remettant en question les stratégies conventionnelles de sécurité alimentaire, à savoir mondialisation et privatisation (et même si la démarche a procédé d'une nécessité et non d'un choix), Cuba a, en pratique, abattu les barrières politiques qui semblent freiner l'adoption de l'éco-agriculture durable » (Annuaire du PNUE, 2009). Ces projets, qui permettent bien évidemment dans un premier temps l'approvisionnement en denrées alimentaires, dévoilent finalement une évolution du rôle de la ville, et en conséquence l'apparition de nouvelles formes du vivre ensemble. 
L'agriculture urbaine semble donc avoir envahi la majeure partie de l'espace des grandes agglomérations, mais y prolifère sous différentes formes, et répond en fin de compte à différents besoins. En effet, en reconfigurant l'espace urbain et son rôle dans la société, l'agriculture urbaine s'est imposée comme un espace d'échanges, un lieu de consolidation du sentiment d'appartenance à la communauté et un territoire où se mêlent productions agricole et culturelle.

En outre, les problèmes alimentaires devenant une préoccupation de plus en plus importante pour les États et les populations, il est possible que l'expérience cubaine et les projets conjoints menés sous l'égide d'organismes internationaux ou d'ONG (re)donnent à l'Île une place de choix sur la scène politique mondiale. En somme, les événements historiques contemporains ont permis à Cuba d'exploiter de nouvelles pistes de la Révolution.

Bibliographie :

ANNUAIRE DU PNUE. 2009. Avancées scientifiques et développements, Nairobi, UNEP/ Earthprint.

COSECHA URBANA. 2004. Memoria y Declaración : la integración de la agricultura urbana en el desarrollo sostenible de las municipalidades, Lima, Centro Internacional de la papa.

CRUZ, M. C. 2004. « ¿Pobreza o desarrollo sostentable? », dans F. Houtart (sous la dir. de), Globalización, agricultura y pobreza, Quito, Abya-Yala, p. 235-250.

CRUZ, M. C. 2005. « El desarrollo urbano sustentable y la agricultura urbana en Cuba. El caso de la ciudad de La Habana », dans H. Avila Sánchez (sous la dir. de), Lo urbano-rural, ¿nuevas expresiones territoriales?, Cuernavaca, Universidad Nacional Autónoma de México, p. 325-354.

DOUZANT ROSENFLED, D. 1996. « L'approvisionnement alimentaire à Cuba: les producteurs entre l'Etat et le marché », dans M. Bey (sous la dir. de), L'agriculture paysanne et la question alimentaire, Lausanne, Fondation pour le progrès de l'Homme, p. 69-70.

ELHAGE SCIABBALA, N. HATTAM, C. 2003. Agricultura orgánica, ambiente y seguridad alimentaria, Rome, FAO.

FARINELLI, B. 2008. L'avenir est à la campagne : solidarité, proximité, autosuffisance, alternative, Paris, Sang de la terre.

FERNANDEZ, M. 2006. " Estudio de caso: cultivando comunidad, alimento y empoderamiento: jardines urbanos en Nueva York y La Habana », dans A. Cohn (sous la dir. de), Agroecología y la lucha para la soberanía alimentaria en las Américas, New Haven, Yale School of Forestry \& Environmental Studies - International Institute for Environment and Development, p. 114-129. 
FERNÁNDEZ, A. 2008. « Le cinéma cubain de la Révolution (1959-2000) : bilan et perspectives », dans F. Moulin Civil (sous la dir. de), Cuba 1959-2006 : révolution dans la culture, culture dans la révolution, L'Harmattan, Paris, p. 231-241.

HALWEIL, B. NIERENBERG, D. 2007. «Cultivar en las ciudades », dans A. Tibaijuka (sous la dir. de), La situación del mundo 2007: nuestro futuro urbano, Madrid, Icaria, p. 115-144.

HOUBEIN, L. 2011. Outside the magic square : a handbook for food security, Kent Town, Wakefield Press.

KNOX, A. MEINZEN-DICK, R. 2006. "Derechos de propiedad, acción colectiva y tecnologías para el manejo de recursos naturales ", dans J. Gonsalves (sous la dir. de), Investigación y Desarrollo Participativo para la Agricultural y el Manejo Sostenible de Recursos Naturales : Libro de Consulta, Ottawa, International Development Research Center, p. 91-100.

LEPAGE, C. 2009. Vivre autrement, Paris, Grasset.

MACDONALD, J. 2005. La otra agenda urbana : tareas, experiencias y programas para aliviar la pobreza y precariedad en las ciudades de América Latina y el Caribe, Santiago de Chile, UN-CEPAL.

MADELEY, J. 2002. Le commerce de la faim : la sécurité alimentaire sacrifie sur l'autel du libre-échange, Paris, Éditions d'En-Bas.

MARECHAL, G. 2010. Les circuits courts alimentaires : bien manger sur les territoires, Dijon, Educagri.

MOSCOW, A. 2000. « Importance de l'agriculture urbaine pour les jardiniers, leur famille et leur communauté : l'exemple de La Havane, à Cuba ", dans M. Koc (sous la dir. de), Armer les villes contre la fin : systèmes alimentaires urbains durables, Ottawa, Centre de recherches pour le développement international, p. 81-88.

PRORAMME DES NATIONS UNIES POUR L'ENVIRONNEMENT. 2002. L'avenir de l'environnement mondial, Bruxelles, De Boeck Supérieur.

PROGRAMME DES NATIONS UNIES POUR LE DEVELOPPEMENT. 2002. Recursos mundiales - La gente y los ecosistemas : se deteriora el tejido de la vida, Madrid, EcoEspaña. RODRIGUEZ, H. G. 2000. "Agriculture dans le parc métropolitain de La Havane, à Cuba », dans M. Koc (sous la dir. de), Armer les villes contre la fin : systèmes alimentaires urbains durables, Ottawa, Centre de recherches pour le développement international, p. 89-94.

ROUX, M. 1997. Cuba, Paris, Karthala. 